\title{
Developing optical efficiency through optimized coating structure: biomimetic inspiration from white beetles
}

\author{
Benny T. Hallam, ${ }^{1, \star}$ Anthony G. Hiorns, ${ }^{1}$ and Peter Vukusic ${ }^{2}$ \\ 'IMERYS Minerals Limited, Par Moor Centre, Par Moor Road, Par, Cornwall PL24 2SQ, UK \\ ${ }^{2}$ School of Physics, University of Exeter, Exeter EX4 4QL, UK \\ ${ }^{*}$ Corresponding author: Benny.hallam@imerys.com \\ Received 7 April 2009; accepted 7 May 2009; \\ posted 26 May 2009 (Doc. ID 109777); published 8 June 2009
}

\begin{abstract}
The recent discovery of brilliant whiteness in ultrathin beetle scales indicated the availability of significant whiteness, brightness, and opacity from limited sample thickness. This is achieved in the beetle through optimization of the packing density of scattering centers in its elytral scales. Here, we directly test and apply this idea to whiteness and brightness in the production and appearance of mineral coatings on paper by varying the scattering center parameters that underpin its optical properties. Through biomimetic design principles, we find that desirably high optical scattering from mineral coatings can be achieved. Commercially, by using appropriately designed coating formulations, this leads to the prospect of equal optical performance using less scattering material. () 2009 Optical Society of America

OCIS codes: $\quad 160.1245,260.0260,290.5850,350.4990$.
\end{abstract}

\section{Introduction and Background}

Whiteness is a concept that carries considerable emotive power. Successful marketing campaigns have managed to convince us that brilliant whiteness is associated with increased purity, quality, freshness, and cleanliness-making whiteness synonymous with enhanced brand value. In recent work [1], the Cyphochilus spp. beetle was shown to exhibit ultrabright whiteness arising from random arrangements of cuticular filaments that occupy the interior of the scales that cover its body (Fig. 1). Although these scales are only $5 \mu \mathrm{m}$ thick, their whiteness, brightness, and opacity values were experimentally measured to be significantly high when compared with synthetic coatings made from mineral particles of a similar refractive index. This represents a combination of properties that are commercially exploitable.

0003-6935/09/173243-07\$15.00/0

(C) 2009 Optical Society of America
Currently, many technologies use mineral coatings to enhance the whiteness, brightness, and opacity of surfaces and materials. Indeed, a broad range of minerals and mineral-based surface coatings underpin the visual appearance and optical performance of a great many devices, commercial products, and everyday objects that are designed to appear white. One of the largest uses of white mineral by volume is in the manufacture of paper. For this, mineral inclusions are used in the body of the paper and also often as mineral coating layers. The function of this is to define the appearance of the paper and to control the interaction of printing inks with the substrate. White paper's visual appearance results from a combination of factors that primarily comprise the shape and size distribution of the scattering particles in the paper's mineral coating [2-9]. This affects the ability of the paper surface to scatter light and, in the absence of any absorption effects, to generate the whiteness, brightness, and opacity of the paper sheet. The light absorption coefficient, a result of the imaginary component of the mineral's complex refractive index also plays an important role in the overall optical 

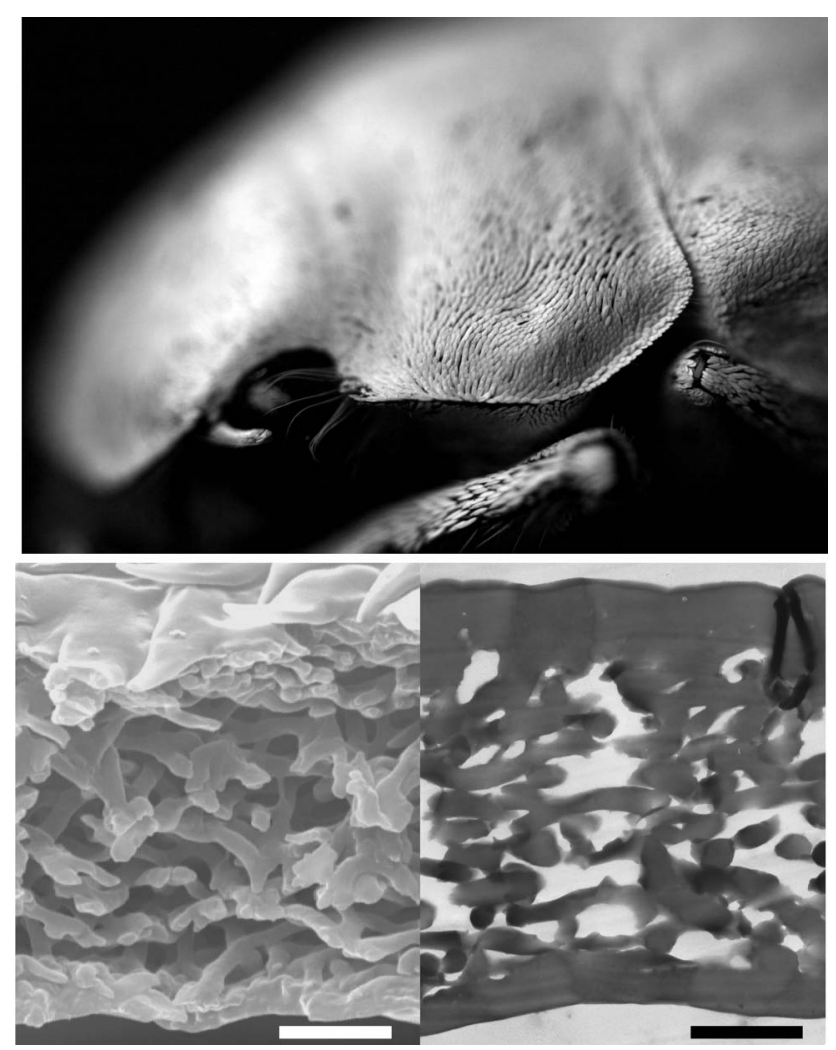

Fig. 1. (a) Head, legs, and body of the Cyphochilus beetle are covered with flattened scales, the interiors of which comprise a random network of interconnected filaments shown here in (b) scanning electron microscopy (SEM) and (c) transmission electron microscopy (TEM) images [Scale bars: (a) $5 \mathrm{~mm}$; (b) and (c) $1 \mu \mathrm{m}]$.

experience. However, this tends to be carefully controlled through crude selection and beneficiation techniques that remove colored impurities or reduce their effectiveness.

In practice, the minerals most commonly used in paper coating formulations are ground calcium carbonate (GCC), precipitated calcium carbonate (PCC), and kaolin. These are typically formulated with a latex binder and other thickening agents to produce a water-based colloidal slurry that may be coated onto the paper surface at high speed $(1800 \mathrm{~m} / \mathrm{min}$ is typical on modern coating machines) to produce an even mineral coating layer thickness of approximately $10-40 \mu \mathrm{m}$ thick depending on the end application. Unlike gloss paints, the mineral component of the formulation is well above the critical pigment volume concentration (cPVC) [10], leading to a dry structure in which the mineral particles pack together to form a mineral matrix that surround a network of airfilled pores or voids. It is the refractive index contrast between mineral and air that enables light scatter to occur. By careful control of the mineral particles, the size and spacing of the scattering centers formed by those pores [11-15] may be controlled. Optimization of paper appearance therefore becomes a balance between the size, number density, and separation of those air pores.
Optimization of the appearance of Cyphochilus beetles has been achieved through natural selection. The constraints associated with the insect's developmental morphology and the optical properties of its scales' constituent materials have dictated the form and structure of the light scattering system that makes up the scales. It is the random network of interconnecting cuticular filaments within each scale that produces such highly efficient optical whiteness and brightness. In particular, the filament width and the packing density are the key variables in creating its optimally tuned appearance [1]. Similarly, from a technological perspective, conventional wisdom might suggest that the optimization of mineral coating layers should also center on defining the optimal size of the scattering unit. In the mineral coating matrix, it is the air voids that become discrete units surrounded by a matrix of solid GCC, PCC, or kaolin material. In practice, however, for minerals with equal size distribution widths, making larger air pores requires larger particulate constituents of the solid material. A consequence of this is that it leads to a lower number density of scattering sites since there will be a larger mineral separation between adjacent air pores.

An alternative approach to optimization is to compromise on the ideal size of the scattering unit and instead target the largest number density of scattering centers. In practice this means reducing the separation between coating pores. If this is reduced too far, however, it can introduce the problem of "optical crowding," where adjacent scattering centers no longer act as discrete entities, but interact with the incident radiation as paired, or composite systems [16-18]. This leads to a reduction in the scattering efficiency. A further problem is that the resultant pore size will be far from optimum since the reduction in separation is achieved by making the constituent particles of the mineral matrix smaller.

The beetle scale system appears to limit the effect of optical crowding by ensuring a suitable spacing between the intrascale scattering filaments. In practice this is achieved by decoupling the inter-relationship between the size of the fibril and that of the air void. Although these fibrils are randomly arranged in a 3D interconnecting network, an indication of the interfibril spacing is available via transmission electron microscopy (TEM) images of scale cross sections [Fig. 1(c)]. Analysis of the $50 \mathrm{~nm}$ thick sections, an example of which is shown in the TEM image in Fig. 1(c), indicates a mean spacing between the filament portions of $0.60 \pm 0.26 \mu \mathrm{m}$ (where the error stated is the standard deviation over all measurements and is due to variation in the size and shape of the filament segments presented in the TEM section). Analysis of TEM images suggests an average fibril width of $250 \pm 50 \mathrm{~nm}$ (again with the error represented by the standard deviation over all measurements). This contrasts with an average wood pulp fiber, which has a fiber width of approximately 

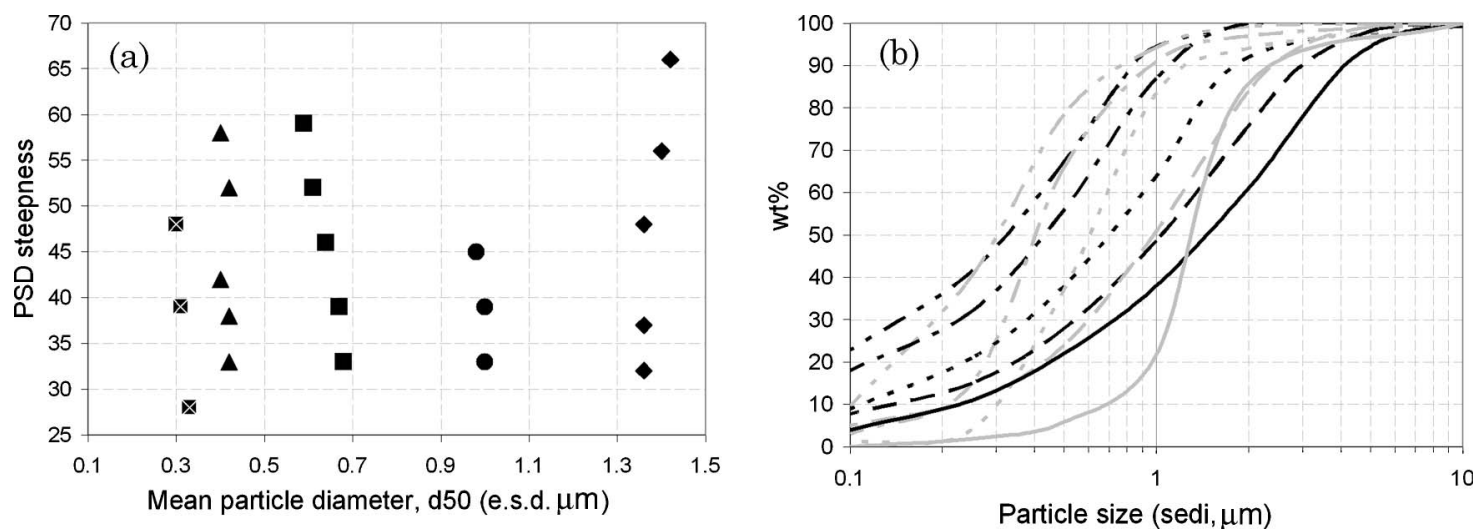

Fig. 2. (a) Matrix of pigments coated in the study, allowing the effect on coating structure of changes of D50 and PSD steepness $(\mathrm{D} 30 / \mathrm{D} 70 * 100)$ to be studied independently; (b) blending broad (black) and narrow (gray) distribution pigments with matched D50 enabled the matrix of coating minerals to be controlled.

$10-36 \mu \mathrm{m}$ for hardwoods and up to $50 \mu \mathrm{m}$ for softwoods.

The combination of variables associated with scattering centers in this beetle's scales appears to be the key to its ultraefficient whiteness and brightness. For the investigation described in this paper, we have applied this doctrine to mineral coatings by systematically varying the key parameters that describe the constituent minerals. This enables an experimental examination of the impact these system variables have on the structure of the resultant mineral coating matrix. Finally, we investigate how this structure relates to the optical performance of the finished coated paper. We also investigate the conditions under which optical crowding begins to degrade optical scattering. The results indicate that a compromise between the size, number density, and spacing of the scattering centers is required to optimize scattering performance.

In dimensionally-isometric particle systems, a constituent mineral may be characterized according to its physical attributes such as the coarseness of the median particle size (referred to as its D50) and the width of the associated particle size distribution (PSD). In this work we have developed model mineral particle sets with strictly controlled D50s and PSDs, and have coated them onto base paper substrates. Through suitable blends of carbonates with broad and narrow PSDs and equal D50s, an extensive set of mineral additive system parameters has been investigated. This has enabled a detailed evaluation of the effect of PSD width and D50 magnitude on scattering to be evaluated while other mineral additive parameters remained constant.

\section{Experimental Details}

Calcium carbonate mineral additives (industrially referred to as nonabsorbing pigments) with broad and narrow PSDs were selected so that they had near-equal D50 values. Particle size measurements were conducted by measuring sedimentation with time via x-ray attenuation on a Sedigraph 5100 . Through appropriate blending of partner pairs of these pigments, a wide range of PSD widths [Figs. 2(a) and 2(b)] was made accessible for use in the optical scattering experiments. A simple measure of PSD width is the PSD steepness. This is defined as the gradient on a log plot of the cumulative distribution of the mass of the blend's particles, calculated at one standard deviation about the median point (D50). In practice, this may be calculated as described in the caption underneath Fig. 2.

The pigments were formulated with $10 \mathrm{pph}$ (parts per hundred on mineral content, by weight) styrene-butadiene latex binder and 0.5 pph carboxymethyl cellulose thickener to make the coating formulation. A Helicoater was used to apply a range of mineral coating weights for each coating formulation at $800 \mathrm{~m} / \mathrm{min}$ onto a $52 \mathrm{gm}^{-2}$ wood-free base using a steel blade. To make the results more applicable to paper coating applications, some of the coated papers were pressed (calendered) at controlled temperature and pressure.

To remove any variations due to testing conditions, the calendered and uncalendered papers were left to equilibrate at $50 \%$ relative humidity and $23^{\circ} \mathrm{C}$ for 24 h. Their optical characteristics were measured using an Elrepho 3300 spectrophotometer with D65/10 illumination. The air-filled pores of each calendered and uncalendered coating layer were characterized with a Pascal 140/240 mercury porosimeter with appropriate corrections for compression of the mercury, glassware, and oil phase [19].

\section{Results}

\section{A. Optimizing the Size of Scattering Units}

Calculation of the average light-scatter contribution from each individual void is achieved by assuming that the pores within the mineral matrix form the principal light-scatter units in the coating layer. This assumption is valid for reasons well described in the literature [11-15].

By plotting the individual light-scatter contribution $[20,21]$ from individual pores as a function of pore size, the optimal discrete pore size may be revealed. Figure 3 shows the relationship between 
the measured light scatter per pore from each different mineral system and leads to conclusive evidence that the optimal void size for individual pores is approximately $425 \mathrm{~nm}$ for these material systems.

To compare the experimental data in Fig. $\underline{3}$ with theoretical predictions, the light backscattere $\bar{d}$ from discrete air voids in a matrix of solid calcium carbonate was calculated using Mie theory [22,23]. The air voids were modeled as having a lognormal distribution of sizes about the average pore size. The calcium carbonate was modeled with a refractive index of 1.57, and the incident illumination was set to $460 \mathrm{~nm}$. The scattered radiation was calculated as a function of collection angle and the portion backscattered into the observer's half-space was integrated and plotted. This modeling represents only light that was scattered back toward the observer and would hence constitute the reflected signal, assuming only single scatter interactions. There is excellent agreement with theory on the particle size associated with maximum light scatter per pore; i.e., for pores of $425 \mathrm{~nm}$ diameter. There is good but less precise agreement, however, between experiment and theory $\left(R^{2}=0.94\right)$ for pore diameters below this value. This imperfect agreement for pore diameters smaller than the value of maximum scatter is not unexpected. The Mie model assumes each pore is appropriately spaced from its neighbors so that they act as independent entities, and also assumes that no multiple scattering events occur. Neither of these assumptions is representative of the real system. A more complete description of the complex scattering structure may be obtained using alternative optical models such as those based on discrete-dipole-approximation (DDA) analysis [24], but this has not been attempted in this work.

Previous work [12] has shown that a maximum in light scatter for narrowly-cut particle size fractions of

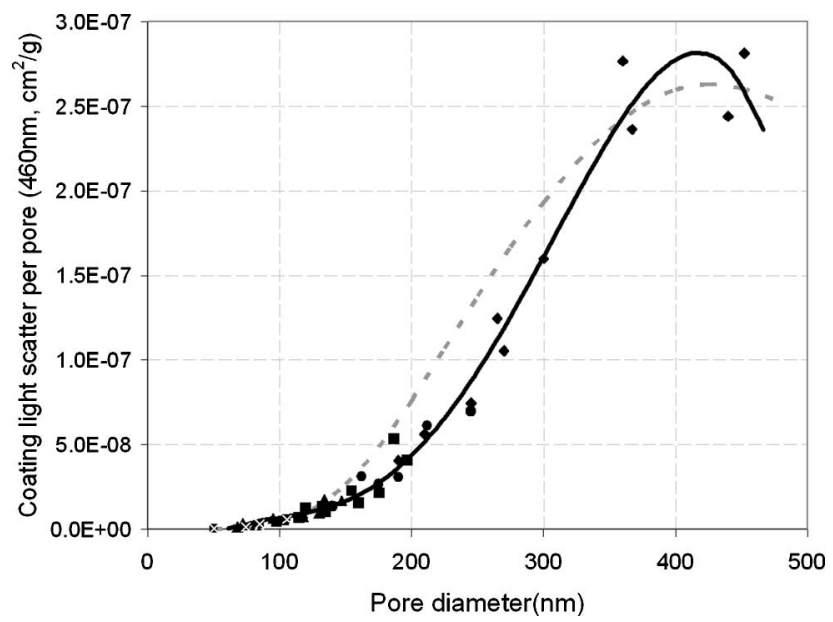

Fig. 3. Light-scatter contribution per air void; data points show experimental data [D50 $=0.3 \mu \mathrm{m}$ (black square with white cross), $\mathrm{D} 50=0.4 \mu \mathrm{m}$ (triangle), D50 $=0.6 \mu \mathrm{m}$ (square), D50 $=1.0 \mu \mathrm{m}$ (circle), $\mathrm{D} 50=1.4 \mu \mathrm{m}$ (diamond)], solid black line is included as a guide to the eye, dotted gray line is the predicted response according to Mie Theory (scattered intensity, arb. units). kaolin give a peak in light scattering for pore diameters between 300 and $440 \mathrm{~nm}$ depending on incident wavelength. A separate study, also with kaolin films [25], showed that voids in the range 300 to $700 \mathrm{~nm}$ gave the largest contribution to light scatter. Both these studies give broad agreement with the current work.

\section{B. Optimizing the Number Density of Scattering Units}

The individual pore size, however, is only one variable that contributes to the scattering. To achieve the relatively large, independent pore diameters highlighted as optimal in Subsection 3.A, a mineral with a large D50 and a narrow PSD was used. This inevitably led to a lower number density of scattering centers since there was greater spacing between pores as a result of the larger physical dimensions of the separating mineral. The manifestation of this is shown in Fig. 4; the number density of pores [26] clearly diminishes with increasing individual pore diameter.

The pore size required for most efficient scattering, namely a pore diameter of $425 \mathrm{~nm}$ (taken from Fig. $\underline{3}$ ), is associated with a low number density of scattering centers. To optimize a system for highly efficient scattering, it is clearly important to reach a compromise between these two competing effects, namely a suitably large pore size and a suitably large number density of pores. This is not a problem in the case of the beetle, since Nature has divorced the link between the size of scattering center and separation between adjacent scattering centers by enabling the scattering element to be grown in the form of fibril. This enables the beetle to simultaneously optimize the size of its scattering center (fibril width) and the fibril separation.

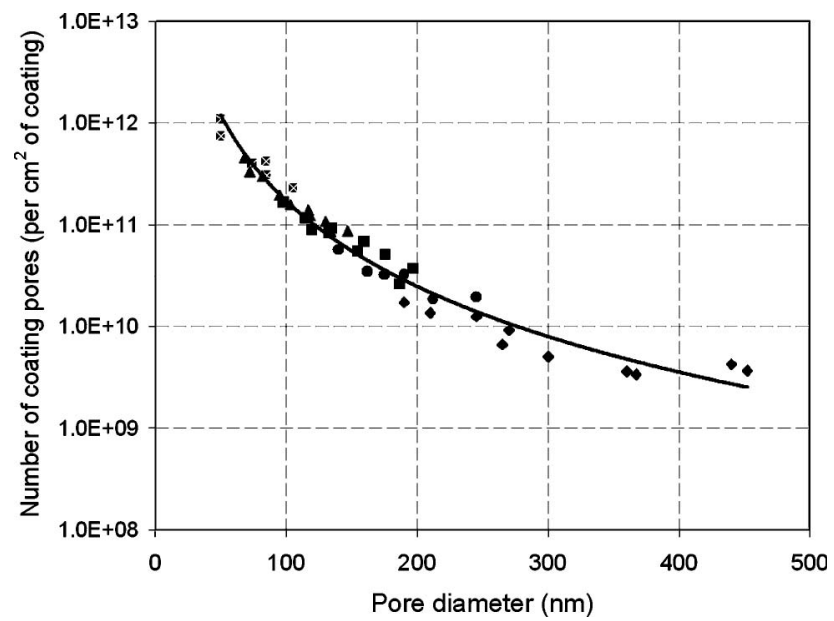

Fig. 4. Number density of scattering sites as a function of average scatter site diameter. Data points show experimental data [D50 $=0.3 \mu \mathrm{m}$ (black square with white cross), D50 $=0.4 \mu \mathrm{m}$ (triangle), D50 $=0.6 \mu \mathrm{m}$ (square), D50 $=1.0 \mu \mathrm{m}$ (circle), D50 $=1.4 \mu \mathrm{m}$ (diamond)], solid line is a guide to the eye. 


\section{Optimizing Separation of Scattering Units}

It is also essential to consider the detrimental effect on scattering efficiency of the influence of optical crowding [16-18]. This occurs when two or more particles are spatially close enough to act effectively as one scattering center. By appropriately modeling the pore structures of our fabricated coatings, it is clear that the average separation between their pores decreases rapidly as their number density increases (Fig. 5). This is an entirely expected phenomenon as it is the mineral comprising the surrounding matrix that controls both as interdependent parameters.

To better understand the importance of optical crowding in scattering experiments in general and in paper coatings in particular, a complimentary exercise to that described earlier was also undertaken. Blends of $\mathrm{TiO}_{2}$ (DuPont RPS Vantage) with a solid latex sphere (Dow 722HS) were formulated and coated onto base paper in different blend ratios. The plastic sphere was carefully selected to ensure that it had a D50 that closely matched that of the $\mathrm{TiO}_{2}$ (average D50 $=0.42 \pm 0.03 \mu \mathrm{m}$ ). Since the latex was almost monosized, and the $\mathrm{TiO}_{2}$ has a narrow PSD, the system may be considered as a good approximation to a set of monosized spheres, in which different blend ratios are characterized by greater or lesser displacement of one species by another. To remove any associated scattering due to air voids within the coating layer (which might be indistinguishable from the $\mathrm{TiO}_{2}$ related scattering) the coated papers were immersed in a suitably mixed index-matching fluid. This was done to optically match the latex spheres and leave the $\mathrm{TiO}_{2}$ particles in an otherwise homogenous medium. The fluid was a blend of mineral oils, carefully mixed to minimize any scattering from the fluid-saturated sheet that was coated with $100 \%$ latex. The point of minimum reflection occurs when the fluid fills the pore structure in the paper coating and is optically-matched to the coating particles. The different blend ratios then effectively act to provide different spacing for the $\mathrm{TiO}_{2}$ particles in a controlled manner according to the particle volume fractions. The experimental data showing light-scatter contribution from indi-

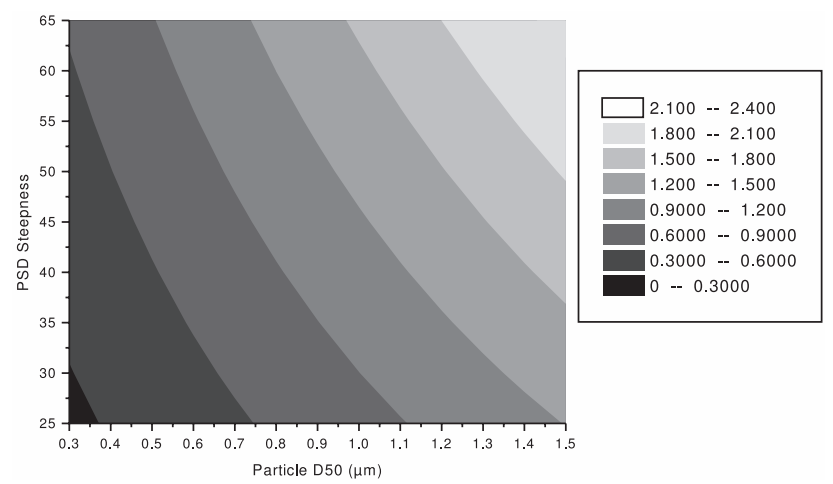

Fig. 5. Average pore separation as a function of typical mineral parameters. vidual $\mathrm{TiO}_{2}$ particles as a function of average adjacent particle separation are presented in Fig. 6 .

The experimental data in Fig. 6 reveal that when the separation between individual $\mathrm{TiO}_{2}$ particles becomes too small, the light-scatter contribution from individual particles diminishes due to optical crowding. The point at which this occurs is for particle separations (surface-to-surface) of less than approximately $0.5-0.9 \mu \mathrm{m}$ depending on incident wavelength. This distance is the equivalent of approximately 1.1 free-space wavelengths in the middle of the visible waveband $(550 \mathrm{~nm})$. This corresponds to approximately 1.7 wavelengths within the fluid-filled paper coating as the wavelength becomes compressed by the material refractive index, assumed to be $n=1.57$.

This value is approximately $10 \%$ higher than theoretical modeling reported elsewhere [16], which suggests that the onset of optical crowding occurs when the surface-to-surface distances between particles falls lower than the wavelength of light within the medium. Comparing this result with the data presented in Fig. 5 suggests that only systems with narrow PSD and large D50 will be free from some degree of optical crowding. Obtaining good light scatter from such systems requires a balance to be struck between the degree of optical crowding and the large number of scattering pores available.

The beetle structure comprises $250 \mathrm{~nm}$ fibrils separated by air voids with an average width of $0.60 \mu \mathrm{m}$. It is interesting to note that our investigations suggest that this is the near exact spacing for $\mathrm{TiO}_{2}$ particles at which optical crowding occurs in the center of the visible waveband. This suggests that the spacing of individual fibrils in the beetle structure is designed to maximize the number density of scattering fibrils without diminishing their efficiency through optical crowding.

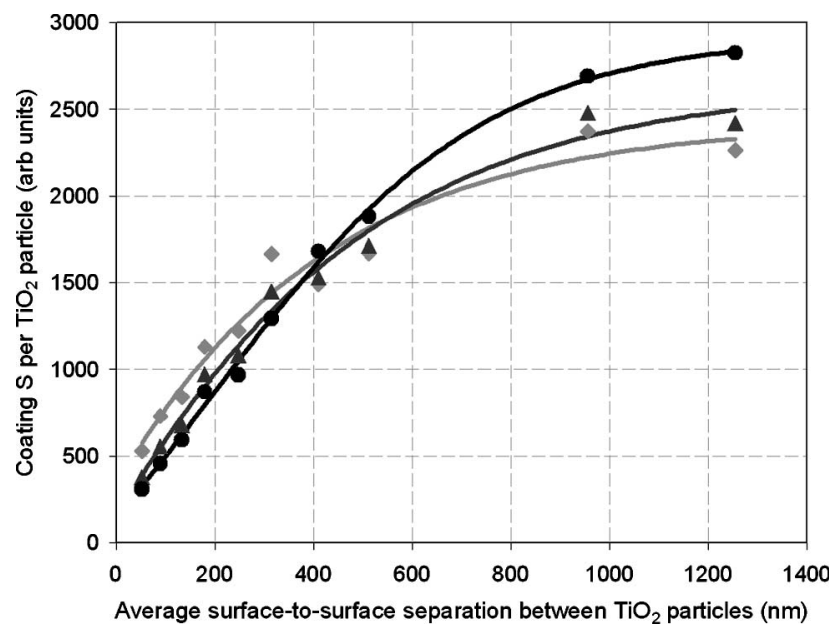

Fig. 6. Light-scatter contribution from individual $\mathrm{TiO}_{2}$ particles as a function of average particle separation for red light at $650 \mathrm{~nm}$ (black), green light at $550 \mathrm{~nm}$ (medium gray) and blue light at $450 \mathrm{~nm}$ light (light gray). 


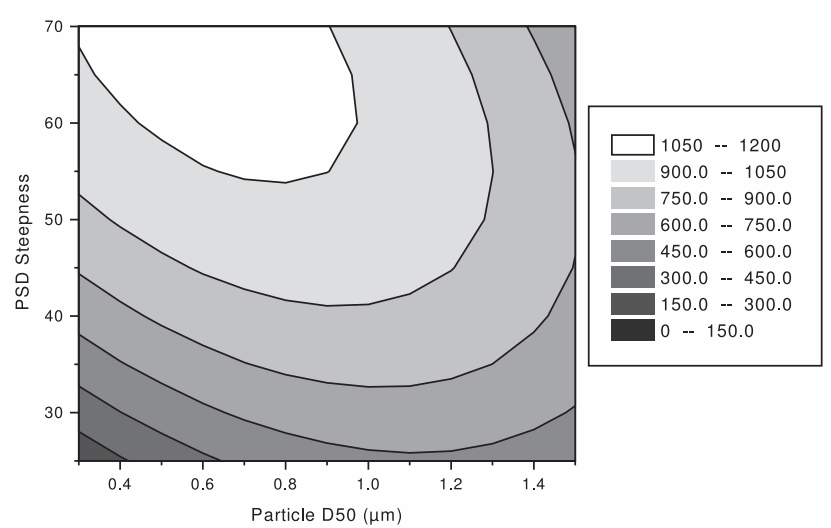

Fig. 7. Calendered sheet light scattering (at $460 \mathrm{~nm}$ ) as a function of typical mineral parameters.

\section{Optimizing the Overall Coating System}

To optimize a full mineral coating system, all the factors described so far must be carefully balanced. Figure 7 shows how the underlying physical mineral parameters affect the final scattering performance.

Figure 7 shows the finished sheet light-scatter potential as a function of mineral D50 and PSD width. The data clearly show the interplay between number density, size, and separation of the scattering units created. There is a cost in terms of light scatter for using particles that are too small in size as the contribution from each individual void becomes so small that it cannot be compensated by the increase in the void number. Likewise, the more optimally sized voids created by the large particles with narrow PSD also suffer from low scattering in the finished sheet. This is due to the low number density of voids.

It is interesting to note that coatings comprising $100 \%$ solid latex spheres have been shown elsewhere [27] to provide maximum light scatter with particle diameters equal to $500 \mathrm{~nm}$. This qualitatively agrees with our findings because such systems would represent high PSD steepness (i.e., the particles are essentially monosized). The reason that narrow PSD mineral systems give greater light scatter than broad PSD mineral systems is that greater void volume is generated for a given void-void separation.

Figure 7 also shows that the optimum D50 for light scatter tends to shift depending on PSD steepness. This demonstrates that there is a complex relationship between these parameters. A possible cause for this is the introduction of ultrafine particles that have a detrimental impact on light scatter by partially filling the air voids and reducing their individual pore volume. It is obvious that the broader the PSD (lower PSD steepness), the more ultrafine particles are introduced into the system. It is logical, therefore, that the PSD must move to coarser D50 to maintain the void volume required for optimal light scattering.

\section{Conclusions}

In this investigation, lessons learned from a previous study of ultrawhiteness in Cyphochilus spp. beetles have been used to examine the parameters underpinning light-scatter generation in biomimetically inspired mineral coating layers on paper. The interplay between the size, number density, and separation of the scattering units in these coatings has been confirmed to play a pivotal role in the optimization of light scatter and has been quantified in detail. The best performance was produced from an overall compromise between all these interdependent parameters, which themselves have been intrinsically linked to specific mineral parameters, such as the D50 and PSD that are routinely controlled in industrial mineral processing during manufacture. These processes themselves do not currently allow accurate replication of the random multiply interconnected fibril system of the Cyphochilus beetle scale interiors on a large scale. However, they have allowed us to evaluate the specific optical design ideas that underpin this beetle's ultrawhiteness and have enabled mineral processing design parameters to be mapped in a systematic manner to enable optimization.

To move outside the system constraints examined in the current work (i.e., that corresponding to dimensionally-isometric scattering centers) it is necessary to consider multimineral solutions where more optimized pore sizes, located at smaller void separations, may be investigated. Such systems might conceivably be accessed by, for example, mixing narrow-PSD carbonate with platy kaolin. This would preserve the large void volume but might reduce the void separation by virtue of being highly anisometric. This is the subject of further investigation.

The authors are grateful to Matthew Cheeseman and Ian Soper for technical assistance. We also thank the board of Imerys Minerals Limited for permission to publish this work. Peter Vukusic gratefully acknowledges funding from the Royal Society.

\section{References}

1. P. Vukusic, B. Hallam, and J. Noyes, "Brilliant whiteness in ultrathin beetle scales," Science 315, 348-348 (2007).

2. G. Pring, C. Nutbeem, and B. Lindqvist, "New solutions for optical performance in coated papers," presented at the 19th PTS Coating Symposium, Munich, 14-17 September 1999 .

3. C. Nutbeem, J. C. Husband, and J. S. Preston, "The role of pigments in controlling coating structure," in PITA Coating Conference, Bradford, Proceedings (PITA, 2005), pp. 97-102.

4. B. Hallam, C. Nutbeem, and T. Asano, "Optimisation of steep carbonate coating formulations with ultra fine platy kaolin," presented at the TAPPI Coating and Graphic Arts Conference, Miami, Florida, 22-25 April 2007.

5. J. C. Husband and C. Nutbeem, "A practical approach to the use of engineered pigments," Paper Technol. 42, 57-66 (2001).

6. P. Burri, C. Naydowski, D. Spielmann, and E. Swanson, "Using classical theory to design a pigment with improved optical properties," Tappi J. 80, 193-199 (1997).

7. D. W. Donigian, J. N. Ishley, and K. J. Wise, "Coating pore structure and offset printed gloss," Tappi J. 80, 163-172 (1997).

8. F. B. Stieg, "Opaque white coatings," in ACS Symposium Series 285: Applied Polymer Science, 2nd ed., R. W. Tess and G. W. Poehlein, eds. (American Chemical Society, 1985). 
9. R. J. Bruehlman, L. W. Thomas, and E. Gonick, "Effect of particle size and pigment volume concentration on hiding power of titanium dioxide," Off.l Dig. 33, 252-267 (1961).

10. W. K. Asbeck and M. Van Loo, "Critical pigment-volume relationships," Ind. Eng. Chem. 41, 1470-1475 (1949).

11. P. Lepoutre and A. Rezanowich, "Optical properties and structure of clay-latex coatings," Tappi J. 60, 86-91 (1977).

12. L. F. Gate, "Light scattering in microporous materials using a photon diffusion model," J. Phys. D 5, 837-846 (1972).

13. P. Lepoutre and B. Alince, "Effect of post-treatment on the optical properties of plastic pigment coatings," Tappi J. 64, 67 (1981).

14. P. Lepoutre, "The structure of paper coatings: an update," Prog. Org. Coatings 17, 89-106 (1989).

15. L. Abrams, C. W. Favorite, P. J. Capano, and R. W. Johnson, "Using mercury porosimetry to characterise coating pore structure and its relation to coating optical performance," in Proceedings of the 1996 TAPPI Coating Conference (TAPPI, 1996), pp. 185-192.

16. L. E. McNeil and R. H. French, "Multiple scattering from rutile $\mathrm{TiO}_{2}$ particles," Acta Mater. 48, 4571-4576 (2000).

17. F. B. Steig, "Ending the 'crowding/spacing theory' debate," J. Coatings Technol.59, 96-97 (1987).
18. J. H. Braun, "Crowding and spacing of titanium dioxide pigments," J. Coatings Technol. 60, 67-71 (1988).

19. J. Kettle, "Investigation and computer modeling of the pore structure of paper and of consolidated coatings," Ph.D. dissertation (University of Plymouth, 1996).

20. P. Kulbelka and F. Munk, "Ein Beitrag zur Optik der Farbanstriche," Z. Tech. Phys. (Leipzig) 11a, 593-601 (1931).

21. N. Pauler, Paper Optics (Lorentzen \& Wettre, 2000).

22. G. Mie, "Contributions to the optics of turbid media, particularly of colloidal metal solutions," Ann. Physik 330, 377445 (1908).

23. For example, the Mie Theory calculator by Ivan Charamisinau or the Mie Plot v3.4.10s by Philip Laven

24. A. Penttilä, K. Lumme, and L. Kuutti, "Light-scattering efficiency of starch acetate pigments as a function of size and packing density," Appl. Opt. 45, 3501-3509 (2006).

25. N. A. Climpson and J. H. Taylor, "Pore size distributions and optical scattering coefficients of clay structures," Tappi J. 59, 89-92 (1976).

26. R. W. Wygant, R. R. Iyer, J. P. E. Jones, and D. O. Cummings, "Glossing properties of engineered kaolins," in Proceedings of the 1998 TAPPI Coating Conference (TAPPI, 1998), p. 837.

27. J. Borch and P. Lepoutre, "Light reflectance of spherical pigments in paper coatings: a comparison with theory," Tappi J. 61 (2), 45 (1978). 